\title{
Cascading Cache Layer in Content Management System
}

\author{
Apoorva Ganapathy \\ Senior Developer, Adobe Systems, San Jose, California, USA \\ E-mail for correspondence: apganapa@adobe.com
}

\begin{abstract}
Caching involves the temporal storing of data in a separate folder. Cascading is the arrangement of something in sequence from top to bottom. Cascading cache layer in content management system places data in layers and sequence in order of importance. The cached data are also removed based on their order of importance. Caching is majorly about input and output of content and data, this brings the need for cascading management system to make accessing data easier than usual. This work takes a look into caching and how it works. It considers various levels of caching in the content management systems. It tries to explain what cascading is in a content management system as well as its importance. This work explains how cascading cache in layers would make it faster and more efficient to access data.
\end{abstract}

Key words: Cache, Cascading, Layers, Memory, Storage, Buffers, CMS Protocol, Algorithm

\section{INTRODUCTION}

It is pronounced as 'cash.' Cache is utilized for storing information or information for some time in the PC world. It very well may be a software or hardware device. A small amount of over-the-top expensive and quicker memory is utilized to build the performance of often utilized information or last utilized information stored and kept in a rapidly open media storage temporarily. This is the cache customer's nearby storage, and it is unique in relation to the whole storage. Operating Systems, CPU, programs, applications generally use the cache, and they are the regular cache clients. Cache comes in because the primary or whole storage can't deal with the high demands from cache clients. With cache, the ideal opportunity for admittance to information diminishes, and cache likewise shortens inactivity in this manner, improving info and output. Storing expands application preparation and performance since all applications depend on info and output.

There is lot of layers in general for caching various types of files ending with extension .html, .json, .xml, .txt, .pdf, .jpg, .png, .gif and so on. For example, web servers, content delivery networks. The cascading cache layer is a process that caches files and assigns unique keys to the files by adding a file parallel to the cached file. It notes down the order of dependency with other files loaded before this file so that the order is followed during recaching or cache invalidation. The order is followed so that the cache gets deleted based on the references. This would avoid a lot of unnecessary cache invalidations and supports cache re-use, which in turn increases the percentage of a cache hit so that performance of the page remains highly better.

\section{How does CACHE Works?}

At whatever point a customer needs admittance to information, it would initially check the cache base. On checking the cache, if the required information is discovered, it is called 'cache hit.' The measure of attempts that end up as cache hits is known as the hit rate or ratio.

'Cache Miss' the circumstance where the required information isn't cannot be seen in cache database. The required information is then gotten from the virtual memory and placed in the cache at whatever point cache miss happens. The place where the framework performs this assignment depends on the strategy of the framework or the coaching algorithms in it. The algorithm and content choose the technique choosing which information to import, erase and supplant. TLB, an abbreviation for Translation Lookaside Buffer. It can store new translations of virtual memory to actual addresses. The primary spot the framework looks through when a program alludes to a virtual location is the CPU. On the off chance that the necessary memory is as yet missing, the following spot turned upward is the memory's actual location, first checking the TLB. The actual memory is then looked at if the TLB doesn't contain the location. Virtual memories are added to the TLB as they are interpreted. Being on the processor makes a recovery from the TLB quicker, diminishing inactivity. The TLB utilizes the high-running recurrence of present-day CPUs for its potential benefit. 
TLBs support computers that permit various clients with a client mode (confined) and portion mode (unlimited). They use permissions on peruse and compose pieces to empower sharing. Nonetheless, performing multiple tasks can bring about performance issues. This fall in performance level is known as cache waste, and it happens when PC movement doesn't advance because of abuse of assets or reserving framework clashes.

\section{Cache vs. buffer}

The two buffers and cache fill in as a temporary holding place for data. The two of them additionally control the in and out the development of data from the holding territory utilizing algorithms. Even though the two buffers and cache temporarily hold data, their explanations behind doing so differ.

\section{Cache versus RAM}

Cache memory and RAM are similar since both of them place information closer to the processor to diminish response time inertness.

On the other hand, a RAM cache typically fuses neverending memory embedded on the motherboard and memory modules that can be presented in specific openings or association zones. The mainboard transport offers induction to these recollections.

Computer chip cache memory is between 10 and 25 times faster than RAM, several nanoseconds to respond to the CPU request. RAM cache, regardless, is faster in its response time than appealing media, which passes on $\mathrm{I} / \mathrm{O}$ at rates in milliseconds.

\section{Importance of cache}

The importance of cache couldn't possibly be more significant. Cache limits dynamic data latency, that is, the measure of time expected to move data from the essential storage to customers. Since frameworks work through info and output data processing operations, the decrease in latency for operational data expands the application's performance.

Caching frameworks redirect information and output to cache. It diminishes output and info undertakings to outside storage and lessens the level is SAN exercises. Data might be kept for all time in the normal storage or disk array. This considers security and assurances are the data through capacities permitted by the array. Instances of such highlights incorporate replication or depictions. A Cost-compelling procedure that can be used to lessen latency is Flash. Flash is generally implied for some piece of the workload. The cache memory might be unique in relation to the fundamental framework memory. It can likewise be set in a chip joined to the framework chipboard. The new generation of machines permits an increment in cache memory by redesigning the central Processing Unit (CPU) and framework board to a later period. In contrast to the more established generations, which give open openings that might be used to grow the cache memory space, the new generation of framework sheets doesn't offer that.

\section{Cache Algorithms}

These are orders, directions, and programming on the manners in which the cache ought to be kept up. Illustration of cache Algorithms are:

Least frequently used (LFU): Analysis of the rate a passage is gotten to. It eliminates the data with the least check first.

Least Recently Used (LRU): this masterminds data got to recently near the highest point of the cache. After the cache storage gets full, the LRU data gets erased.

Most Recently Used (MRU): this erases data most recently got to first. This strategy works best when more established data are fundamental and more prone to be gotten to.

\section{Cache Policies}

Write-around this approach includes processing days to storage, keeping away from the cache. It holds the cache back from being overpowered during enormous traffic of data information and output. The drawback of this strategy is that data should be perused straightforwardly from storage alone. This implies that the perusing operation would turn out to be moderate in light of the fact that the data isn't in the cache.

Write-through cache: this strategy permits new data to be written in both cache and storage. This is a benefit since it will empower a speedy read of recently entered data as they are cached. Processing data isn't finished until they are written in both cache and storage. This is an impediment since it can because latency recorded as a hard copy operation.

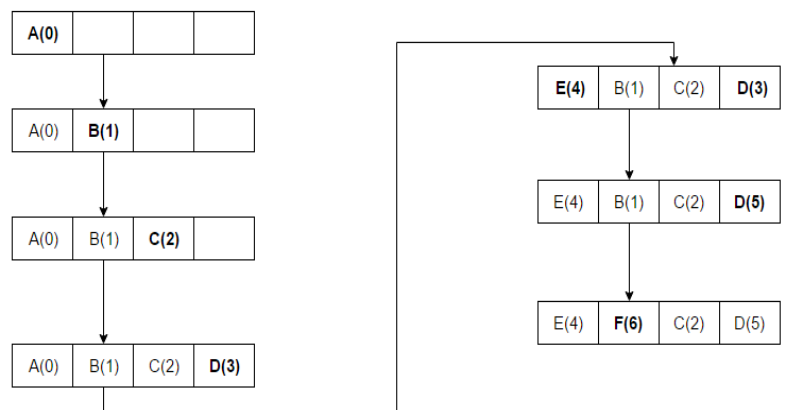

Figure 1: cache policy (Source: en.wikipedia.org)

\section{How CACHE ARE USED}

Cache server: Cache server: this network is a committed server that goes about as a web server. It saves data or site pages locally. Cache servers are otherwise called intermediary cache. 
Disk cache: stores recently read data and here and there another data design with more odds of getting to soon. There are various kinds of disk caches and some cache data utilizing how frequently it is perused. Often read storage blocks are called hot squares and are naturally kept in the cache.

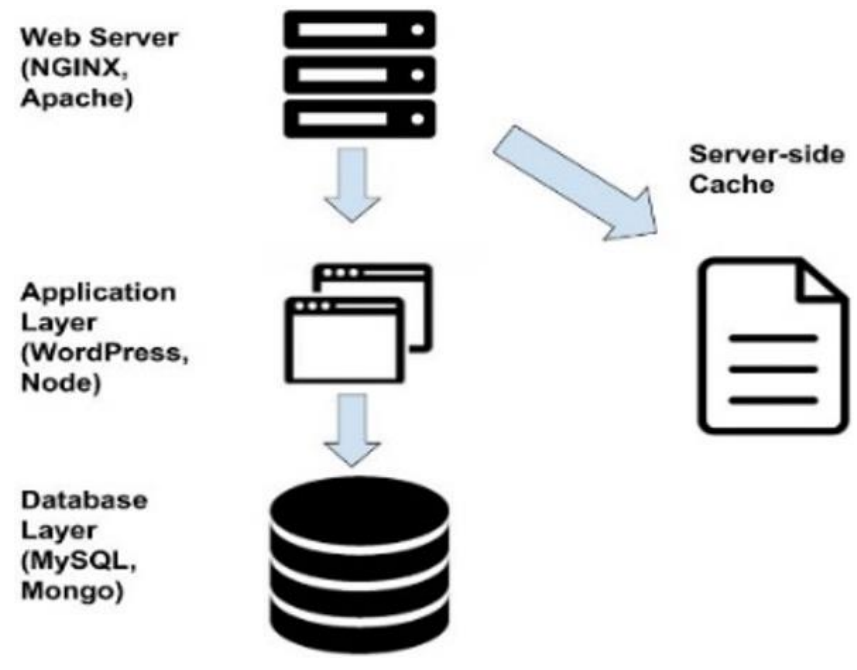

Figure 2: server-side-cache (Source: jeffreyeverhart.com)

Cache memory, while a few sorts of RAM (Random Access Memory) permit microprocessors access quicker than other normal RAM. Cache storage is typically attached to the CPU and used for caching directions that are constantly gotten to. A RAM cache is significantly speedier contrasted with a disk-based cache. In any case, cache memory is quicker than a RAM cache as it is nearer to the Central Processing Unit (CPU).

At whatever point you enter a website or site page, you send a solicitation, and the documents mentioned might be stored in the figuring storage of your internet browser's cache. Chrome, Firefox, windows explorer all increment the degree of use performance by utilizing program cache.

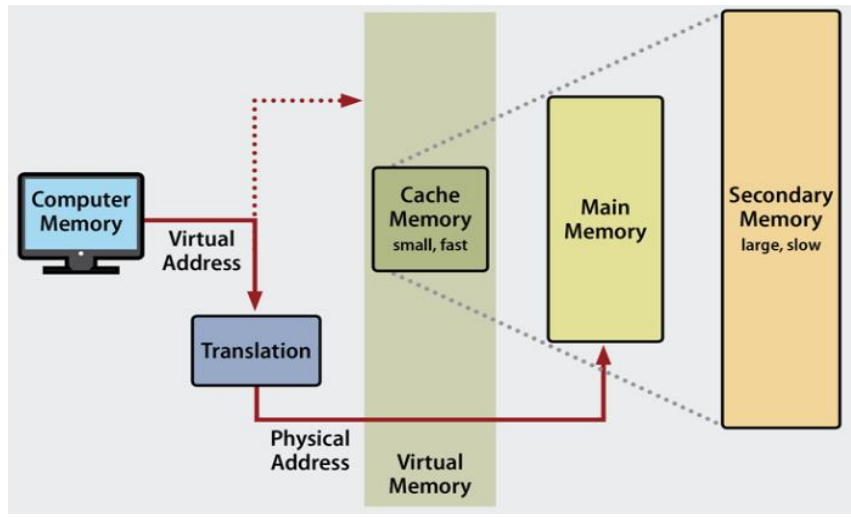

Figure 3: Virtual-Memory (Source: Vivadifferences.com)

Utilizing the back key to return the keep going page you were on permits the program to gather most of the data it needs from the cache instead of getting them sent back from the web server once more. This method is alluded to as an 'understood cache.' The cache is more open to a program than site pages and servers. This makes it quicker for the program to recover and peruse data from the program cache than peruse or rehash from website pages.

Persistent cache: This is seen as the main storage capacity. Computer reboot or when the system crashes content here won't be lost. Backup batteries are used to protect data, and data is moved to a battery-enabled Dynamic RAM (DRAM) as added protection from data loss.

\section{Cache LeVel}

Cache storage levels are the hierarchy of memory. Computers have different storage levels. They include:

Level 1-L1

Level 2-L2

Level 3-L3

Level 4-L4

The cache memory storage is arranged according to the speed and the size of the cache.

\section{Level 1-L1}

This is the quickest memory available in a computer system. This cache storage level houses the data that the CPU will most likely use in completing a particular job, i.e., in terms of priority. Cache Level 1 size depends on the computer. So highly advanced consumer Computer currently enables a 1MB Level 1 cache. However, these are pretty expensive and are still scarce and far between. Intel's Xeon and some other server choosers also enable a 1-2MB L1 memory cache. Level 1 cache sizes are not fixed. To know the exact size of the L1 memory cache size, you will have to check the system properties and specifications before purchasing.

The L1 cache is mainly divided into two sections: the command cache and the data cache. The command cache handles the information on the tasks which the CPU must perform. While the data cache holds the data upon which the task is to be done.

\section{Level 2-L2}

This is a much slower cache than the level 1 cache. The size is usually bigger than L1. While a Level 1 cache may be in kilobytes, the recent generation of L2 memory caches would be in megabytes. E.g., AMD's highly rated Ryzen 4 $5600 x$ has a 384 Level1 cache, 3MB level 2 cache and a 32MB level 3 cache.

Level 2 cache size differs, and it depends on the type of CPU. The size of level 2 caches is usually between $257 \mathrm{~KB}$ to $8 \mathrm{MB}$.

A lot of recent computers pack above $257 \mathrm{~KB}$ L2 cache, although this is a small size. Furthermore, most of the highly advanced modern CPUs usually have a larger L2 memory cache above $8 \mathrm{MB}$ 
Regarding speed, the Level 2 cache comes behind the L1 cache, but it is, however, faster than the normal system RAM. The level 1 memory cache is normally super advance and faster than the system RAM, while the L2 cache is not as much, just around 26 times faster.

\section{Level 3-L3}

The third storage level of cache, in earlier times, was usually seen on motherboards. However, this was a long time ago, during the period when the majority of CPUs had merely single-core processors. Currently, the modern third storage level cache can be big, with highly advanced client CPUs enabling L3 caches up to 32MB. Some may go as high as $64 \mathrm{MB}$.

The L3 cache is the biggest, but it has the slowest cache storage unit. Now, CPUs enable level 3 cache in the CPU. Level 3 cache is more knowledge to be on the general memory pool that the entire chip can make use of. However, levels 1 and 2 caches exist on each core on the chip itself.

\section{Level 4-L4}

Level 4 cache is accessible, and it allows dynamic sharing of memory between the Central processing unit (CPU) and Graphics processing unit (GPU).

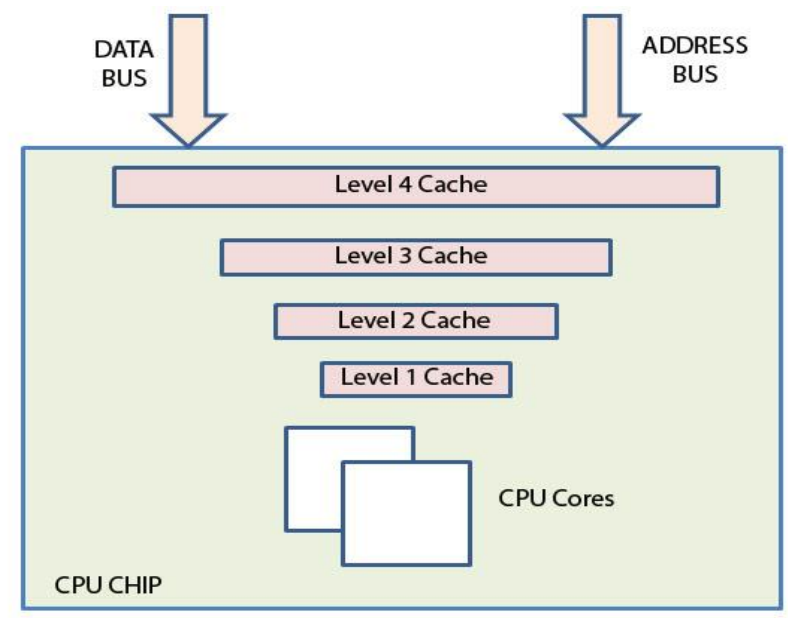

(c) teach-ict.com

Figure 4: Cache-hierarchy (Source: www.teach-ict.com)

\section{LAYERING}

Layering in system programming is organizing programs to fit into separate networking layers. Software scripts divides the layers into several parts for easy access. Some protocols divide the layers into five (5). Some other protocols add more than five layers. There are lots of layers in general for caching various types of files with extensions. Normal networking hardware and software are separated into five layers. Some have more than five layers, however.

System software engineers must have the ability to program software that interacts well with themselves.
Simple interaction across the web involves placing messages down from one layer to another. The messages will flow from the originating layer up and through the same route back (peer layers).

In more complicated interactions, messages are passed through middle correspondence channels Called routers. At every router, messages are left behind through layers and afterward proceeded onward down while in transit to an alternate router or destination system.

Through layering, each layer has a unique purpose and function for communication. A programmed system defines the function of each layer. The protocol sets the order and format for messages transferred between the interacting systems and the actions that should be taken. Communicating systems are often customers or processes in need of a service and a server. Protocols are usually scripts for encoding and decoding data. Many web application programs chose specific scripts that customers may use in navigating the cache servers.

\section{The Physical layer}

This layer encodes bits into transmission mediums. The bits are encoded into multiple media (fiber, wire or radio broadcast) through different channels to increase sensitivity. Each PL program identifies content, blueprint for encoding, and a blueprint that starts \& end bits. When the many interacting systems share one media, it becomes the responsibility of the physical layer to detect collisions.

\section{The Application Layer}

This layer oversees the correspondence among clients and server cycles and peers the cycles offering administrations to one another. A few programs exist relying upon the sorts of system management.

\section{The transport layer}

This layer handles the transmission of data amongst processes on multiple system servers on the internet. There are multiple protocols in this layer.

\section{The network layer}

The network layer conveys data among the various frameworks on the web. This enhances the movement issue for the other layer. If you send an email across the network, the network layer sends social events of data from your framework to the framework on which your mail server programming runs.

Utilizing the network layer system, the transport layers at the two closures just need to manage sending information to and gathering it from measures on their particular system - your mailer convention in your system or at the mail server from the opposite side.

The network layer determines the medium used by blocks of data through the internet. The route is adjusted in the internet protocol (IP) to use links with lesser traffic. 


\section{The data link layer}

This layer delivers data and information from across a particular link. The main issue this layer solves is coordinating interaction through a connected medium. Different communications entities in the Ethernet group of protocols can be connected via a hub (a connected tool that only sends data from each interacting device that it is connected to.

This causes the need for systems to detect other communicating systems to enable them to communicate and connect.

This makes it necessary for cache systems to have a mechanism that will identify interacting entities in other for communication to be supported between any pair of entities. A coordination tool becomes necessary to deal with clashes that may come up due to systems sending data simultaneously.

\section{Cascading Cache layers}

Cascading's in a real sense, implies organizing something in an arrangement or sequence. Cascading is utilized to make and execute complex information processing work processes on a Hadoop group using any JVM-based language like Java and others, concealing the hidden intricacies.

Consider cascading like a waterfall. A waterfall cascades down a bluff or gorge or wherever its peak is, with the highest point of the waterfall by an enormous being where the most water is streaming.

Cascading cache layer has a similar type of priority, with style in the most critical importance having the most relevance, etc., down the line, in a cascading fashion. Everything under of that acquires what was previously set, except if expressly redefined.

The cascading cache layer is a sequence that caches files, yet in addition appoints unique keys to the files by adding a document corresponding to the cached scrape taking note of down the order of reliance with different files stacked before this record, so that during re-caching or cache invalidation, the charge is followed, so the cache gets erased dependent on the references. This would stay away from a lot of pointless cache invalidations and support cache re-use, thus increasing the level of a cache hit, so the page's performance remains exceptionally better.

Different content management systems execute a few layers of caching to ensure quick content altering and perusing encounters. These caching layers cover significant content management objects, including page HTML, media content, customer resources, configurations, etc. Cascading CMS can store the cache items in the web server memory, on a circulated cache data store, or the customer (program) and empowers you to configure the caching components to convey ideal performance coordinating your desired use case. Customer cache headers can be controlled through cascaded CMS to calibrate the duration a content thing stays in the program cache. You can give intermediary servers guidelines on how to store content and convey it quicker. Content delivery network (CDN) is wholly supported out of the crate for page HTML, JavaScript, CSS, Fonts, and media content (for example, pictures, recordings, archives). Server Output cache execution in the CMS ensures that content is served in a split second from memory instead of being processed without fail. Application-level caches deal with storing data that set aside a more prolonged effort to measure (for example, DB questions, configuration files, and so on) and serving it from the web server memory.

A conceivably hazardous workflow was perhaps cascading a help and endeavoring to create a layered cache. The layered cache component settles on numerous decisions for each layer at different degrees and resolutions. Few out of every odd specialist co-op can stay aware of the requests required to construct a layered cache at the speed the cache system requires.

\section{REAson FOR SEPARATING LAYERS}

In making a site, the construction needs to be consigned to your HTML, scripts. A portion of the likely gains of segregating the layers are:

- Shared resources: When you compose an outside CSS or JavaScript report, any page on the site can use that record. If you need to improve that archive, perhaps to revive some typographic styles on the site, each page that utilizes that template will change. There is no convincing motivation to change each site's page, which could be a tedious undertaking for a huge site.

- Faster downloads: After the content or layout has been downloaded by your client for the headliner, it is saved by the web program. Since these common assets are as of now contained in the program store, different pages referenced in the program load even more rapidly, which improves page speed and execution.

- Multi-specific parties: when there are more than an individual chipping away at a site quickly, utilize different control frameworks that honor records for being taken a gander at and guarantee that everyone works with the latest changes. This alliance is basically harder to do if styles and practices are weaved with structure reports.

- SEO: A website showing an exact detachment style and construction will in all probability perform better for web records. They can crawl that content all the more sufficiently and grasp the page without getting ruined in visual style and conduct data.

- Accessibility: External configurations and content documents are more open to people and projects. For 
instance, programming creators can oversee content from the construction layer all the more successfully without managing styles that they can't use notwithstanding.

- Backward likeness: An engineered site with independent advancement layers is more dedicated to being backward attainable in the light of how projects and gadgets that can't use certain CSS styles or have JavaScript harmed can, notwithstanding, see the HTML. You would then have the decision to upgrade your site logically with highlights for the projects that help them.

\section{CONCLUSION}

When you cache data, you store them away in special and different storage. It is stored temporarily on the device and allows for fast and efficient system application and browser speed. The cache keeps the data on the device. The files could be photos, videos and other kinds of files.

The cascading cache layer is a system protocol and programming that allows you to cache many different files ending with extension in layers. Each layer holds a particular type of file. Cascading cache layer assigns special keys to the files to make them traceable in layers and sequence. It notes the order of dependency of the files flowing from the top like an actual Cascade. This order is followed during caching, and re-caching and the cached files are also deleted based on this order and reference.

The benefits of having a cache in managing your content on the web servers and content delivery networks cannot be over-emphasized. Caching increases the amount of time needed to access and transfer data from main storage to clients.

Also, with cascading cache content management system, files arranged in a sequence makes it easier to get to files and remove the file in the order of importance.

\section{REFERENCES}

Ganapathy, A. (2015). AI Fitness Checks, Maintenance and Monitoring on Systems Managing Content \& Data: A Study on CMS World. Malaysian Journal of Medical and Biological Research, 2(2),

113-118. https://doi.org/10.18034/mjmbr.v2i2.553

Ganapathy, A. (2016). Speech Emotion Recognition Using Deep Learning Techniques. ABC Journal of Advanced Research, 5(2), 113-122. https://doi.org/10.18034/abcjar.v5i2.550

Ganapathy, A. (2017). Friendly URLs in the CMS and Power of Global Ranking with Crawlers with Added Security. Engineering International, 5(2), 87-96. https://doi.org/10.18034/ei.v5i2.541

Neogy, T. K., \& Paruchuri, H. (2014). Machine Learning as a New Search Engine Interface: An Overview. Engineering International, 2(2), https://doi.org/10.18034/ei.v2i2.539

Paruchuri, H. (2015). Application of Artificial Neural Network to ANPR: An Overview. ABC Journal of Advanced Research, 4(2), 143-152. https://doi.org/10.18034/abcjar.v4i2.549

Paruchuri, H. (2017). Credit Card Fraud Detection using Machine Learning: A Systematic Literature Review. ABC Journal of Advanced Research, 6(2), 113-120. https://doi.org/10.18034/abcjar.v6i2.547

Vadlamudi, S. (2015). Enabling Trustworthiness in Artificial Intelligence - A Detailed Discussion. Engineering International, 3(2), 105-114. https://doi.org/10.18034/ei.v3i2.519

Vadlamudi, S. (2016). What Impact does Internet of Things have on Project Management in Project based Firms?. Asian Business Review, 6(3), 179-186. https://doi.org/10.18034/abr.v6i3.520

Vadlamudi, S. (2017). Stock Market Prediction using Machine Learning: A Systematic Literature Review. American Journal of Trade and Policy, 4(3), 123-128. https://doi.org/10.18034/ajtp.v4i3.521

Vadlamudi, S. (2018). Agri-Food System and Artificial Intelligence: Reconsidering Imperishability. Asian Journal of Applied Science and Engineering, 7(1), 33-42. Retrieved from https://journals.abc.us.org/index.php/ajase/article/view/ $\underline{1192}$ 